\title{
Possible suppression of canted spin order in the double-exchange lanthanum manganites
}

\author{
S. K. Mishra and S. Satpathy \\ Department of Physics and Astronomy, University of Missouri, Columbia, Missouri 65211 \\ and Max-Planck Institut für Festkörperforschung, Heisenbergstrasse 1, D-70569 Stuttgart, Germany \\ F. Aryasetiawan \\ Department of Theoretical Physics, University of Lund, Solvegatan 14A, 22362 Lund, Sweden \\ O. Gunnarsson \\ Max-Planck Institut für Festkörperforschung, Heisenbergstrasse 1, D-70569 Stuttgart, Germany
}

(Received 25 October 1996)

\begin{abstract}
The existence of the canted (noncollinear) spin order in the double-exchange magnets is examined by solving a model Hamiltonian using a combination of the Hartree-Fock approximation and an exact diagonalization scheme using the Lanczos method. It is shown that the tendency for the canted spin order depends significantly on the electronic parameters and that the double-exchange mechanism does not always lead to a canted magnetic state, even for small carrier concentration. [S0163-1829(97)00506-7]
\end{abstract}

The striking correlation between ferromagnetism and metallic conduction in the mixed valence oxides $\mathrm{La}_{1-x} \mathrm{Ca}_{x}\left(\mathrm{Mn}_{1-x}^{3+} \mathrm{Mn}_{x}^{4+}\right) \mathrm{O}_{3}$, observed by Jonker and van Santen, ${ }^{1}$ was explained qualitatively by Zener's idea of double exchange. ${ }^{2}$ The double-exchange mechanism was subsequently reformulated and studied in two classic papers by Anderson and Hasegawa ${ }^{3}$ and by De Gennes. ${ }^{4}$ According to this mechanism, both ferromagnetism and conduction are linked to the mobility of the manganese conduction electrons. Taking the example of $\mathrm{La}_{1-x} \mathrm{Ca}_{x} \mathrm{MnO}_{3}$, on which considerable attention has been focused following the discovery of colossal magnetoresistance, ${ }^{5,6}$ the $\operatorname{Mn}\left(t_{2 g}\right)$ electrons constitute the localized spins, while the $e_{g}$ electrons (or holes) constitute the mobile carriers. The motion of these "Zener carriers" couples the localized spins ferromagnetically since this minimizes the kinetic energy, reminiscent of the $\mathrm{Na}$ gaoka state, ${ }^{7}$ where the presence of a hole in an otherwise half-filled Hubbard model turns the entire lattice ferromagnetic. In contrast to the Hubbard model, where the formation of the ferromagnetic Nagaoka state is governed by the Coulomb interaction, in the double-exchange mechanism, the important parameter for the ferromagnetic coupling is the Hund's rule exchange $J_{H}$ between the localized and the conduction electrons.

In addition to the ferromagnetic coupling between the localized spins mediated via the conduction electrons (double exchange), there also exists a superexchange interaction between the localized spins, and the ground-state magnetic structure is determined by a competition between the two. De Gennes ${ }^{4}$ argued that for small Zener carrier concentration, this competition always results in a canted spin order. Experimentally, the presence of a ferromagnetic moment in the manganites and its increase with the Zener carrier concentration $^{8}$ have been suggested as evidence for canting, 4,9 based on which a canted order for $\mathrm{La}_{1-x} \mathrm{Ca}_{x} \mathrm{MnO}_{3}$-type systems in the range of $0.1<x<0.2$ is generally assumed. However, recent experiments have cast doubt on this assumption. For example, the lack of a sharp temperature dependence of the collinear-noncollinear magnetic transition with $x$, contrary to prediction, has led Troyanchuk to suggest the presence of a mixed state, consisting of ferromagnetic and antiferromagnetic (AF) phases, ${ }^{10}$ as opposed to a canted state. Thus the issue of the existence of canting in the manganites is still under debate.

This paper contains two main results: (1) The doubleexchange mechanism does not always lead to a canted magnetic order, even for small carrier concentration $x$ and (2) The electronic parameters in $\mathrm{La}_{1-x} \mathrm{Ca}_{x} \mathrm{MnO}_{3}$ are such that canting may be suppressed in these compounds.

To summarize De Gennes' argument for the existence of canting, consider, for example, a layer cubic structure in which all spins within a (100) layer are aligned ferromagnetically, while they are canted by the angle $\theta$ with respect to spins in the successive layers. This is the canted version of the observed AF type $A$ structure of $\mathrm{LaMnO}_{3}$ considered in this paper. Then for a small Zener carrier concentration $x$, introduced by $\mathrm{Ca}$ doping, the total energy is given by a sum of the superexchange and the band-structure terms,

$$
E=J \cos \theta-[4 t+2 t \cos (\theta / 2)] \times x,
$$

where $x$ being small, all carriers were assumed to occupy the lowest band state. Here $J$ is the AF superexchange between the localized spins on successive planes and $t$ is the nearestneighbor hopping integral between orbitals of same spin. The basic argument of De Gennes was that the loss of the exchange energy is of the second order, while the gain in the kinetic energy is of the first order (in the angle of deviation from the AF order), so that the kinetic energy term always favors a canted order. This can be seen from Eq. (1), which has the minimum for a canted order with $\theta=2 \cos ^{-1}(t x / 2 J)$ for all values of $t$ and $J$, and for $x<2 J / t$. Beyond this $x$, a ferromagnetic order is obtained.

In De Gennes' work, in addition to the simplified kinetic energy term, the Hund's rule exchange $J_{H}$ is taken to be $\infty$ and the Coulomb interaction is ignored. Recent density- 

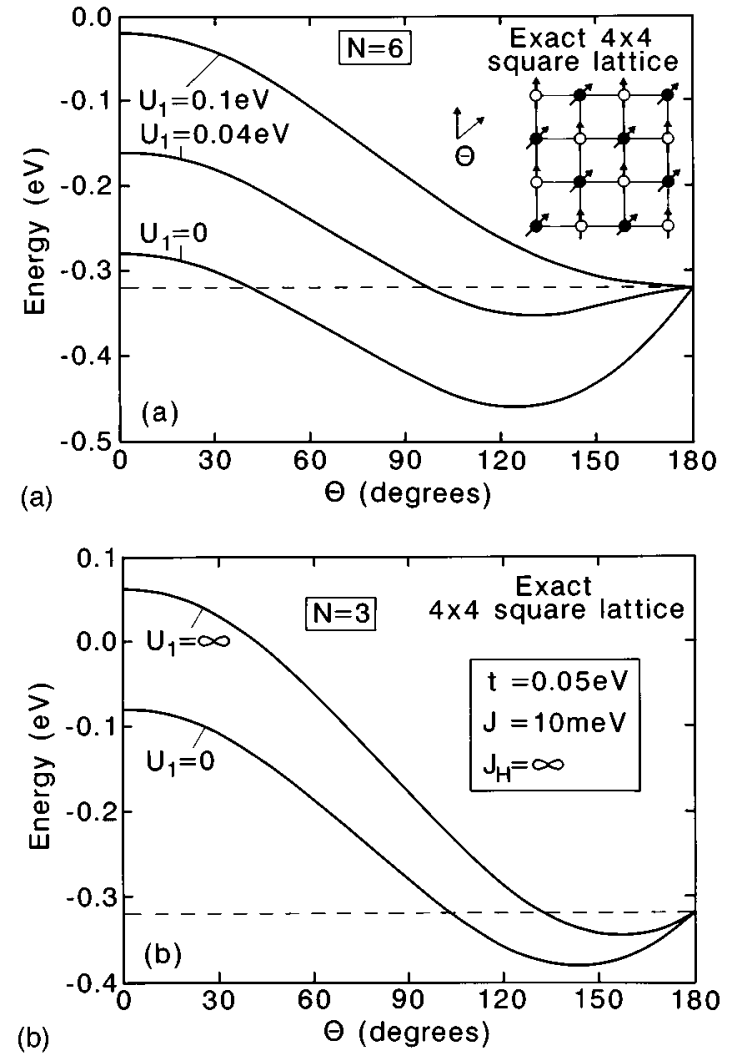

FIG. 1. Energy as a function of the angle of canting $\theta$ obtained from exact diagonalization for the $4 \times 4$ square lattice for two different values of the number of Zener electrons: (a) $N=6$ and (b) $N=3$. The electron parameters used are listed in (b).

functional calculations ${ }^{11,12}$ have shown that these conditions are not satisfied in the lanthanum manganites and, in particular, the exchange splitting is only about twice the bandwidth $W$ of the relevant $e_{g}$ band, i.e., $4 J_{H} / W \approx 2$. As we show below, when these effects are taken into account, the energetics of the canted state are modified, and for appropriate parameters, canting is suppressed.

We consider a model, which is a lattice version of the Anderson-Hasegawa ${ }^{3}$ Hamiltonian but where in addition we have included the Coulomb interaction between the Zener carriers:

$$
\begin{aligned}
\mathcal{H}= & -t \sum_{\langle i j\rangle, \sigma} c_{i \sigma}^{\dagger} c_{j \sigma}+\text { c.c. }+\sum_{\langle i j\rangle} \hat{J}_{i j} S_{i} \cdot S_{j}-2 J_{H} \sum_{i} S_{i} \cdot s_{i} \\
& +U_{0} \sum_{i} \hat{n}_{i \uparrow} \hat{n}_{i \downarrow}+U_{1} \sum_{\langle i j\rangle, \sigma \nu} \hat{n}_{i \sigma} \hat{n}_{j \nu} .
\end{aligned}
$$

The model, restricted to the Mn sites, describes the motion of the conduction electrons (or holes) in the Jahn-Teller split lower $e_{g}$ band (one orbital per site), ${ }^{11}$ in a lattice of localized $t_{2 g}$ spins. The electron-phonon coupling term is not explicitly included in the Hamiltonian, since as argued by De Gennes ${ }^{4}$ it is unlikely to affect the canting energy in any significant way. In Eq. (2), $t$ is the nearest-neighbor hopping integral, $c_{i \sigma}^{\dagger}\left(c_{i \sigma}\right)$ are the creation (annihilation) operators for electrons on site $i$ with spin $\sigma, \hat{n}_{i \sigma}$ is the number operator, $\mathbf{s}_{i} \equiv(1 / 2) \Sigma_{\sigma \nu}\left(c_{i \sigma}^{\dagger} \boldsymbol{\tau}_{\sigma \nu} c_{i \nu}\right)$ is the conduction-electron spin

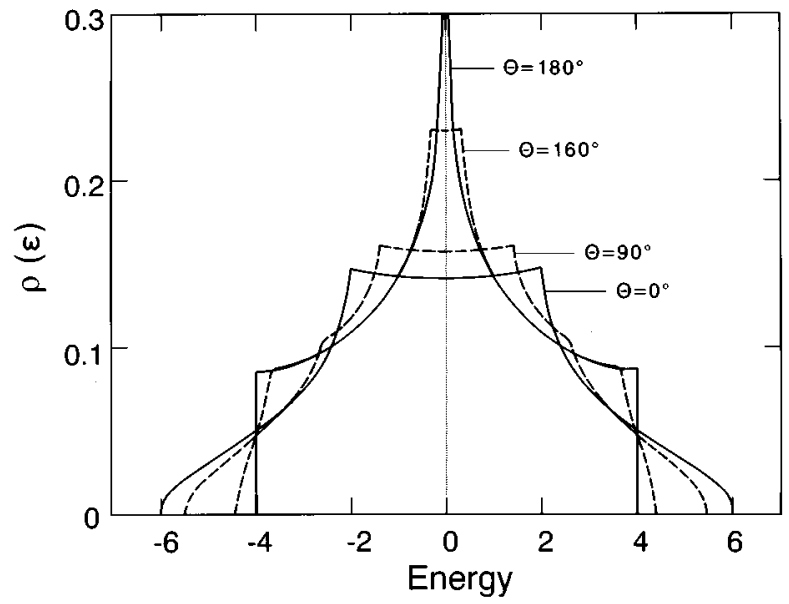

FIG. 2. One-electron density-of-states $\rho(\epsilon)$ for the cubic lattice corresponding to the dispersion relation, Eq. (3). Energy is in units of $t$.

density, with $\tau$ being the Pauli matrices, $J_{H}$ is the Hund's rule exchange, $\hat{J}_{i j}$ is the superexchange between the localized spins $S$, and $U_{0}$ and $U_{1}$ are the on-site and the nearestneighbor Coulomb repulsion terms. Apart from its effect on canting, the Coulomb interaction plays an important role in describing the experimentally observed charge ordering between $\mathrm{Mn}^{3+}$ and $\mathrm{Mn}^{4+}$ ions, ${ }^{13-15}$ an issue we shall discuss elsewhere. ${ }^{16}$ For the present case of one conduction orbital per site, the on-site $U_{0}$ is unimportant if $J_{H}$ is large, since the latter already prevents the double occupancy of a lattice site. However for smaller values of $J_{H}, U_{0}$ is important. Following earlier authors, the $t_{2 g}$ spins are treated as classical, but the correct quantum-mechanical exchange energies are used, so that the energy difference between the parallel and antiparallel alignment of the electron spin with respect to $S$ is given by $(2 S+1) J_{H}$, i.e., $4 J_{H}$ for the present case with $S=3 / 2$. Guided by results of density-functional calculations, typical values of the electron parameters entering into the Hamiltonian are $t \simeq 0.1-0.15 \mathrm{eV}, U_{0} \simeq 8-10 \mathrm{eV}, J_{H} \simeq 1 \mathrm{eV}$, and $U_{1} \simeq 0.2-0.4 \mathrm{eV}$. Without loss of generality, we retain only an effective interlayer nearest-neighbor exchange $\hat{J}_{i j}$, which we estimate to be $J \equiv \hat{J} S^{2} \approx 7 \quad \mathrm{meV} \cdot{ }^{17}$ For $\mathrm{LaMnO}_{3}$, with the $t_{2 g}^{3} e_{g}^{1}$ configuration of the $\mathrm{Mn}$ atom, the $e_{g}^{1}$ conduction band is full, and holes are introduced in this band, one per $\mathrm{Ca}$ dopant, in the mixed compound $\mathrm{La}_{1-x} \mathrm{Ca}_{x} \mathrm{MnO}_{3} .{ }^{11}$

We have studied the ground state of the Hamiltionian (2) as a function of the Zener carrier concentration $x$ and orientation of the $t_{2 g}$ spins using two methods: (i) Exact Lanczos diagonalization on a $4 \times 4$ square lattice and (ii) HartreeFock approximation on the cubic lattice.

To examine the effects of the Coulomb interaction, which affects the canting order by reducing the kinetic energy of the itinerant electrons, we have performed an exact diagonalization study for a $4 \times 4$ square lattice with periodic boundary condition, where the magnetic moments are aligned with a prescribed angle $\theta$ with respect to the nearest neighbors as shown in the inset of Fig. 1(a). The ground state is obtained by a numerical diagonalization of the many-body Hamiltonian using the Lanczos algorithm. ${ }^{18}$ To isolate the effect of 


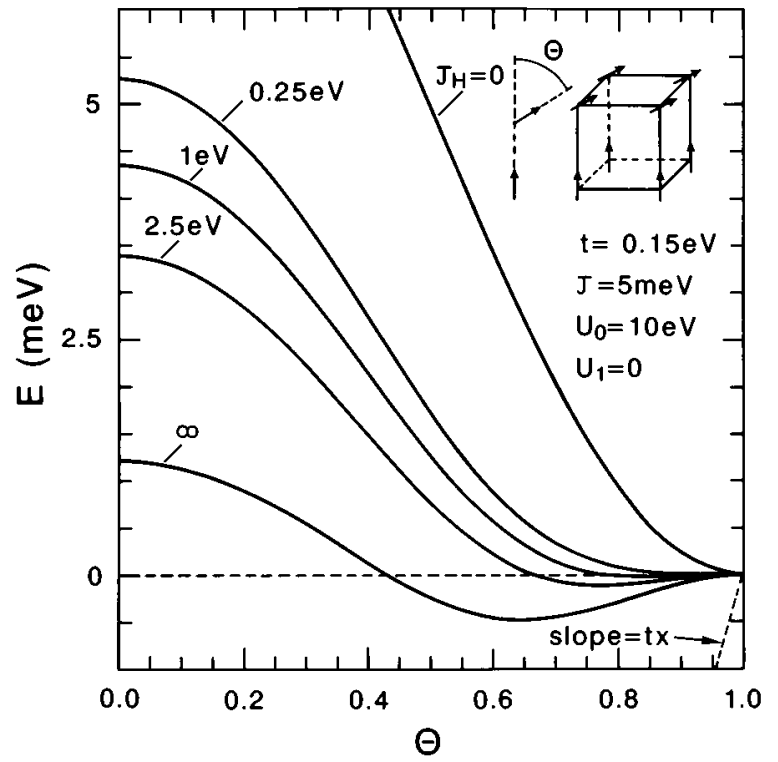

FIG. 3. Total energy as a function of the canting angle $\theta$ (units of $\pi$ ) for the cubic lattice for selected values of $J_{H}$ with Zener hole concentration $x=0.05$. The energy slope corresponding to Eq. (1), $d E /\left.d \theta\right|_{\theta \rightarrow \pi}=t x$, is indicated by the dashed line.

the Coulomb terms, we have taken $J_{H}=\infty$, which makes $U_{1}$ the relevant Coulomb parameter as $U_{0}$ is unimportant in this limit. For $J_{H}=\infty$, the computations are simplified by using a site-dependent axis for spin quantization pointed along the localized spin $S$. Only one spin orientation is then allowed, but now the effective hopping integral becomes $-t \cos (\theta / 2){ }^{3}$

The results are presented in Fig. 1, which shows the variation of the energy with the angle of canting. The minimum of the total energy gives the value of the canting angle, for instance, $\theta \approx 130^{\circ}$ for the case $N=6$ and $U_{1}=0$. The Coulomb interaction $U_{1}$ reduces the kinetic energy as the Zener

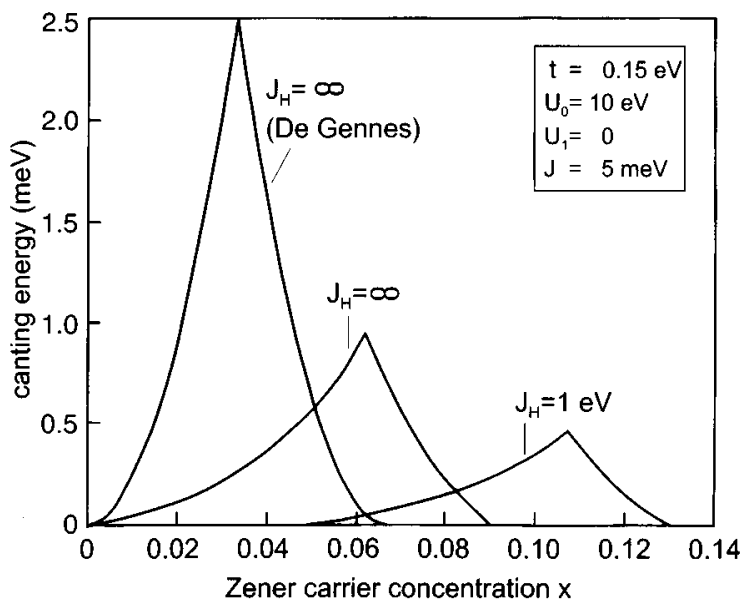

FIG. 4. Variation of the canting energy with Zener carrier concentration for the cubic lattice with layer magnetic structure. Shown also are the results corresponding to De Gennes' expression, Eq. (1). The other two curves correspond to the results of the HartreeFock calculations. The relative values of the canting energies indicate the diminishing tendency towards the formation of a canted spin order as $J_{H}$ is lowered.

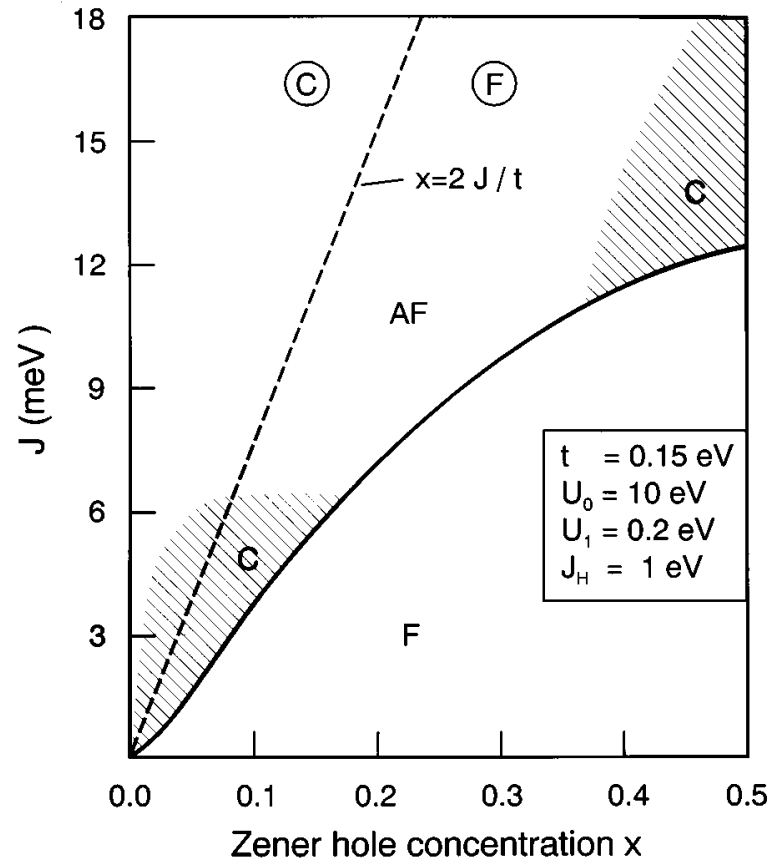

FIG. 5. Dependence of the ground-state magnetic structure on the carrier concentration $x$ and the exchange interaction $J$, for electronic parameters typical for the manganites. Hatched regions indicate ground states with canted order. The dashed line separates the canted and the ferromagnetic regions (indicated by circled $\mathrm{C}$ and $\mathrm{F}$ ) according to Eq. (1), which neglects several effects as discussed in the text.

electrons are forced to move in a correlated manner in order to avoid the simultaneous occupancy of nearest-neighbor sites in the lattice. If the carrier concentration is sufficiently high, then there may exist a critical value of $U_{1}$, beyond which the kinetic energy is sufficiently reduced that the energetics are in effect controlled by the superexchange term $J$. This is seen for the case $N=6$ [Fig. 1(a)], where the tendency towards the canted order is gradually diminished with increasing $U_{1}$, with the canting eventually suppressed for $U_{1} \gtrsim 0.1 \mathrm{eV}$. For smaller carrier concentration, however, the effect of $U_{1}$ is not as dramatic, since the carriers rarely encounter one another. This is illustrated in Fig. 1(b) for the case of $N=3$, where even an infinite value of $U_{1}$ is unable to suppress canting, although, the canting energy is reduced, reflecting the kinetic energy reduction.

The energetics of the canting order with respect to the AF-type $A$ order for the cubic lattice, relevant for $\mathrm{La}_{1-x} \mathrm{Ca}_{x} \mathrm{MnO}_{3}$, were studied in the self-consistent HartreeFock approximation. All Brillouin-zone summations were performed with $\sim 50000 \vec{k}$ points. In the case of the lattice the kinetic energy in De Gennes' expression (1) becomes quickly invalid as $x$ is increased from zero. To illustrate this, we consider the case of noninteracting electrons in the limit $J_{H}=\infty$, so that electron spins are always aligned along $S$ and we have a one-band tight-binding model. The electronic band structure $\epsilon(\vec{k})$ is then given by

$$
\epsilon(\vec{k})=2 t_{\|}\left(\cos k_{x} a+\cos k_{y} a\right)+2 t_{\perp} \cos k_{z} a,
$$

where $\vec{k}$ is the Bloch momentum, the in-plane hopping $t_{\|}=-t$ and the interplane hopping $t_{\perp}=-t \cos (\theta / 2)$. The 
one-electron density-of-states (DOS), $\rho(\epsilon)$, can be expressed in terms of elliptic integrals ${ }^{19}$ and are shown in Fig. 2 for various $\theta$ 's. The case of $\theta=180^{\circ}$ corresponds to an $\mathrm{AF}$ alignment resulting in $t_{\perp}=0$ and therefore to a twodimensional DOS. The DOS near the band bottom $\epsilon_{b}$ is the important part, since this for small $x$ determines the bandstructure energy. If a linear approximation to the DOS near $\epsilon_{b}$ is made, as suggested from Fig. 2, viz., $\rho(\epsilon)=0$ for $\epsilon<\epsilon_{b},(c / 2) \times\left(\epsilon-\epsilon_{b}\right) /(2 t \cos (\theta / 2))$ for $\epsilon_{b}<\epsilon<-4 t$ $+2 t \cos (\theta / 2)$, and $c$ otherwise, with $c \simeq 0.09 / t$, then for a given value of $x$, one finds that the gain in band-structure energy for $\theta \rightarrow 180^{\circ}$ is in fact of the second order, viz., $\delta E_{\mathrm{bs}}=(2 / 3) c t^{2} \cos ^{2}(\theta / 2)$ for $x>2 c t \cos (\theta / 2)$, i.e., of the same order in $\theta$ as the exchange energy in Eq. (1), so that De Gennes's argument is not valid anymore. This effect is seen from the full calculation (Fig. 3) where the energy corresponding to $J_{H}=\infty$ differs significantly from the linear slope $t x$, as would be predicted from Eq. (1).

Now, we discuss how the canting energy is affected as $J_{H}$ is reduced from its infinity value. For large $J_{H}$, one can show using second-order perturbation theory that the canting angle is given by $\cos (\theta / 2)=(t x) /\left(2 J+t^{2} / J_{H}\right)$. Thus, as $J_{H}$ is reduced from its infinite value, the system tends more towards the AF order, i.e., the canting is weakened. For $J_{H}=0$, canting is entirely suppressed, for, in this limit, the conduction electrons are no longer coupled to the localized spins and the magnetic order is then governed solely by the superexchange interaction $J$. This gradual reduction and disappearance of the canting order with diminishing $J_{H}$ is seen from the Hartree-Fock results for the layer cubic lattice (Fig. $3)$.

An indication of the tendency towards the formation of a canted order is the robustness of the canting energy, defined as the energy difference between the state with canting order and the competing state with either the $\mathrm{F}$ or the AF order. The canting energy is shown in Fig. 4, from which we see that the energy gained by canting is appreciably reduced in our model. For instance, the maximum canting energy is about $2.5 \mathrm{meV}$ in De Gennes' model, occurring at $x \simeq 0.03$, while we find the maximum value to be only about $0.5 \mathrm{meV}$ occurring at $x \simeq 0.1$.

In Fig. 5, we summarize the ground-state magnetic structure as a function of carrier concentration and the relative magnitude of the exchange $J$, for the electronic parameters appropriate for the lanthanum manganites. Contrary to the De Gennes expression, Eq. (1), which always predicts a canted spin order for small enough $x$, we see that this is not the case anymore. For instance, the canted spin order is not favored for the entire range of concentration, $0<x \leqq 0.4$, if $J \gtrsim 6 \mathrm{meV}$. Near half-filling ( $x \approx 0.5$ ), the reemergence of the canted region seen in Fig. 5 is related to the divergent twodimensional density-of-states at the band center (Fig. 2). However, since the Jahn-Teller splitting of the twofold $e_{g}$ orbital is already small at half-filling, corresponding to $\mathrm{La}_{0.5} \mathrm{Ca}_{0.5} \mathrm{MnO}_{3}$, a two-band model may be more appropriate in this concentration range. The more complex $C E$ lattice structure is experimentally observed for this case. ${ }^{8}$

We note here that our analysis does not preclude the existence of a canted state for the entire family of manganites. Rather, we have shown that the existence of a canted ground state depends on the relative magnitudes of the various electronic parameters. Furthermore, we have demonstrated that the double-exchange mechanism does not always lead to a canted magnetic ground state, even for small carrier concentration, contrary to the prevalent belief in the literature.

We thank R. Pandit for stimulating discussions. This work was supported in part by the Office of Naval Research under Contract No. ONR N00014-95-1-0439.
${ }^{1}$ G. H. Jonker and J. H. Van Santen, Physica 16, 337 (1950); J. H.

Van Santen and G. H. Jonker, ibid. 16, 599 (1950).

${ }^{2}$ C. Zener, Phys. Rev. 82, 403 (1951).

${ }^{3}$ P. W. Anderson and H. Hasegawa, Phys. Rev. 100, 675 (1955).

${ }^{4}$ P.-G. De Gennes, Phys. Rev. 118, 141 (1960).

${ }^{5}$ R. von Helmolt, J. Wecker, B. Holzapfel, L. Schultz, and K. Samwer, Phys. Rev. Lett. 71, 2331 (1993).

${ }^{6}$ S. Jin, T. H. Tiefel, M. McCormack, R. A. Fastnacht, R. Ramesh, and L. H. Chen, Science 264, 413 (1994).

${ }^{7}$ Y. Nagaoka, Phys. Rev. 147, 392 (1966).

${ }^{8}$ E. O. Wollan and W. C. Koehler, Phys. Rev. 100, 545 (1955).

${ }^{9}$ G. Matsumoto, J. Phys. Soc. Jpn. 29, 614 (1970).

${ }^{10}$ I. O. Troyanchuk, Sov. Phys. JETP 75, 132 (1992).
${ }^{11}$ S. Satpathy, Z. S. Popović, and F. R. Vukajlović, Phys. Rev. Lett. 76, 960 (1996).

${ }^{12}$ W. E. Pickett and D. J. Singh, Phys. Rev. B 53, 1146 (1996).

${ }^{13}$ J. B. Goodenough, Phys. Rev. 100, 564 (1955).

${ }^{14}$ K. Knizek, Z. Jirak, E. Pollert, and F. Zounova, J. Solid State Chem. 100, 292 (1992).

${ }^{15}$ Y. Tomioka, A. Asamitsu, Y. Moritomo, H. Kuwahara, and Y. Tokura, Phys. Rev. Lett. 74, 5108 (1995).

${ }^{16}$ S. K. Mishra, R. Pandit, and S. Satpathy (unpublished).

${ }^{17}$ I. Solovyev (private communication).

${ }^{18}$ E. Dagotto, Rev. Mod. Phys. 66, 763 (1994).

${ }^{19}$ T. Morita and T. Horiguchi, J. Phys. Soc. Jpn. 30, 957 (1971). 